\title{
Les discours sur la diversité culturelle occultent des guerres d'intérêts
}

\section{Bernard Dagenais}

\section{(2) OpenEdition}

12 Journals

Édition électronique

URL : http://journals.openedition.org/communicationorganisation/2757

DOI : 10.4000/communicationorganisation.2757

ISSN : $1775-3546$

Éditeur

Presses universitaires de Bordeaux

Édition imprimée

Date de publication : 1 novembre 2002

ISSN : 1168-5549

Référence électronique

Bernard Dagenais, "Les discours sur la diversité culturelle occultent des guerres d'intérêts », Communication et organisation [En ligne], 22 | 2002, mis en ligne le 27 mars 2012, consulté le 19 avril 2019. URL : http://journals.openedition.org/communicationorganisation/2757 ; DOI : 10.4000/ communicationorganisation. 2757

Ce document a été généré automatiquement le 19 avril 2019

(c) Presses universitaires de Bordeaux 


\title{
Les discours sur la diversité culturelle occultent des guerres d'intérêts
}

\author{
Bernard Dagenais
}

1 Pourquoi réfléchir sur le phénomène de la mondialisation et de ses effets pervers, puisque le problème existe depuis la nuit des temps ? L'histoire de l'humanité est composée de rencontres interculturelles. Le peuplement de la Terre s'est réalisé par les déplacements de population. Les vainqueurs ont imposé aux vaincus langues, coutumes et manières de faire.

2 On parle aujourd'hui de mondialisation et de globalisation comme s'il s'agissait d'un phénomène nouveau. Les quatre grandes religions mondiales, le christianisme, l'islam, le judaïsme et le bouddhisme ont pratiqué depuis des siècles une forme certaine de globalisation, entraînant avec elle les mêmes effets pervers que l'on reproche actuellement au libéralisme économique.

3 Depuis des décennies, à partir de quelques grands centres urbains ou de quelques grandes entreprises et organisations, certains acteurs politiques, marchands, religieux ou sociaux vont dicter au monde entier, avec un discours unique, ce qu'il faut penser, ce qu'il faut manger, comment il faut se divertir et même quel parti politique il faut soutenir.

4 Ce qu'il y a de changé, ce n'est pas tant le phénomène lui-même, quoiqu'il se soit amplifié de façon accélérée au cours du dernier demi-siècle, ce sont les conséquences appréhendées de cette situation et surtout le nouveau discours qui l'entoure. Pour Hotier (2001), si tant de recherches portent sur l'interculturel, c'est sans doute en raison des dangers que la mondialisation des affaires fait peser sur les cultures.

5 L'histoire des grandes civilisations révèle qu'elles ont toujours eu des visées expansionnistes et impérialistes. Les pays européens colonisateurs se sont comportés sur tous les continents conquis comme de véritables barbares alors qu'ils affirmaient apporter la civilisation. Aujourd'hui, parce qu'ils sont les plus puissants du monde, les Américains exportent la notion de mondialisation et de globalisation, de libre circulation des biens et des services, d'ouverture des frontières et de partage global de la culture internationale, des droits et libertés pour tous. Pour eux, la terre est un immense marché 
qui doit s'ouvrir au commerce américain. À ce titre, ils refusent toute distinction entre les biens culturels qui témoignent de l'âme profonde de chaque peuple et les productions de masse culturelles que les Américains savent si bien offrir comme la quintessence du savoir humain.

Les frontières politiques, culturelles ou commerciales existent bel et bien, mais elles sont poreuses de partout. Aujourd'hui chaque peuple fait face à deux options: son appartenance à la collectivité mondiale et ses racines profondes et tribales. S'interroger sur les rapports entre la propension à l'uniformité et l'expression de la diversité, entre la globalisation et l'identité locale, entre le think globally et act locally, c'est en quelque sorte se demander si l'histoire réelle de notre civilisation n'a pas fait fausse route. Aujourd'hui, aucune frontière ne résiste à la radio, à la télévision et à Internet. La mondialisation s'est construite petit à petit et aujourd'hui est devenue une réalité historique incontournable. À l'intérieur comme à l'extérieur des frontières de chaque pays se pratique un apport interculturel inévitable. La question n'est pas de savoir s'il est souhaitable ou pas, mais comment le gérer.

\section{Les religions}

7 Les questions du tchador ou du crucifix dans les écoles publiques ont fait l'objet de nombreux débats. Maintenant, le débat se déplace vers le kirpan. S'agit-il d'un symbole religieux ou d'une arme blanche ? Le problème s'est posé au début de 2002 dans une école de Montréal, au Québec, lorsqu'un jeune homme de religion sikh a voulu porter cet attribut religieux. L'administration scolaire a décrété que c'était une arme. Les parents de l'enfant ont retiré l'enfant de l'école et affirmé qu'il s'agissait d'un grave accroc à la Charte des droits et libertés. Un arbitre a cru trouver la solution : le kirpan pourrait être porté sous les vêtements et à l'intérieur d'une gaine cousue. Les autorités scolaires ont décrété qu'une arme blanche peu importe où et comment elle était portée demeurait une arme blanche.

8 La question des religions suscite un double questionnement : celui de l'immigration et celui des droits et libertés de tous et chacun. L'interculturel habite désormais la cité de façon irrévocable. Mais alors qu'autrefois, c'était les grandes puissances qui exportaient leur religion, parfois de façon brutale, aujourd'hui, elle importe celles des autres de façon lente et irrémédiable.

Les grandes religions se comparent aux multinationales et pratiquent une forme de globalisation que l'on a tendance à oublier. Elles possèdent leurs lieux sacrés, leurs leaders mondiaux et leurs ramifications dans tous les coins de la terre. Mais Robertson (1992), en s'interrogeant sur le phénomène social de la religion, a aussi démontré la complexité de la mondialisation d'une idée, en l'occurrence la religion, qui, tout en étant régie par une structure hiérarchique mondiale, était fortement ancrée dans les idiosyncrasies nationales.

Si jadis les catholiques ont imposé leurs Croisades, aujourd'hui certains islamistes sont entrés dans une guerre sainte contre les infidèles. Lorsque sa religion veut s'imposer au reste du monde, c'est faire acte de civilisation. Lorsque la religion de l'autre s'immisce chez soi, c'est une provocation... Le discours sur la religion constitue donc le plus profond objet d'interculturalisme et d'incompréhension. En Irlande, la guerre de religion dure depuis plus de 250 ans. 
11 La fatwa que l'Iman Khomeiny a prononcé contre Salman Rushdie illustre bien le problème de fond. Parce qu'il a écrit ce qu'il pensait, Rushdie a heurté la sensibilité de certains islamistes. Des dizaines de personnes reliées à l'édition ou à la traduction de son œuvre seront importunées, blessées ou tuées à la suite de cette offensive. Une guerre sainte était lancée et des milliers de fidèles partout au monde ont voulu venger le blasphème.

\section{Le monde des affaires}

12 Le monde des affaires est confronté de façon quotidienne à l'approche interculturelle. Pour opérer sur un marché mondial, les entreprises vont articuler leurs communications selon deux visions différentes. La première consiste à dire que le consommateur, partout dans le monde, partage les mêmes désirs et les mêmes besoins de base et peut, dès lors, les voir satisfaits par une approche de communication universelle. Pour cette école de pensée, les différences entre pays sont davantage une question d'amplitude que d'orientation. On parle alors de standardisation des messages mondiaux.

13 La deuxième approche consiste à dire que chaque culture est particulière et que les consommateurs diffèrent d'un pays à l'autre et que, de ce fait, il faut utiliser une approche plus respectueuse de chaque culture (Kanso, 1992). Autrement, toute communication mondiale a tendance à niveler et enrayer les cultures nationales.

14 L'approche de la standardisation est contredite par diverses théories qui affirment que l'environnement culturel d'un individu moule sa perception de la réalité et que la négliger amène des incompréhensions même meurtrières. Déjà en 1934, Ruth Benedict présentait un essai devenu un classique sur les particularités culturelles des peuples. Et Philippe D'Iribarne (1989, p.11) écrivait que chaque culture avait «une manière spécifique de relier l'homme à la collectivité et de séparer le bien du mal, le légitime de l'illégitime, ce que l'on respecte, ce qui indiffère et ce que l'on méprise ».

15 Par ailleurs, cette même approche est confirmée par le succès international de certains produits culturels qui envahissent la planète. D'un bout à l'autre du monde, on visionne les mêmes films, on adule les mêmes chanteurs, on s'habille avec les mêmes jeans et on se délecte de la même nourriture rapide. Personne n'oblige les différents consommateurs du monde à se jeter sur les mêmes produits, à suivre les mêmes modes, à partager les mêmes idées. Pour Hotier (2001). « il n'empêche que celui qui impose sa culture et celui qui s'y plie, qui l'accepte, voire qui l'espère, pour toutes sortes de raisons qui ne sont pas seulement financières, sont bel et bien coauteurs, co-producteurs. sinon complices, de ce changement ».

Depuis des décennies, de nombreuses études ont démontré l'attrait et l'intérêt de la publicité globale servie à travers le monde (Elinder 1965, Dunn 1966, Fatt 1967, Sorenson et Wiechmann 1975, Marquez 1979, Levitt 1983, Rutigliano 1986). Le problème préoccupe donc les chercheurs depuis plus de 30 ans. L'exemple le plus intéressant à cet effet est la demande d'américanisation dans la vie japonaise qui a fait l'objet de nombreuses recherches : Yoshino 1975, Belk et Pollay, 1985, Rosenberg 1986, Sekigahama 1988, Fields 1989 et Mueller 1992. Ces études ont d'abord examiné l'ampleur du problème, puis tenté de l'expliquer pour enfin conclure qu'il ne touche qu'une fraction de la publicité japonaise, sans démontrer que l'âme japonaise y a perdu son identité. Pas plus d'ailleurs 
que l'utilisation des lutteurs de sumo japonais dans la publicité occidentale n'influence l'identité des peuples auxquels cette publicité s'adresse (tiré de Dagenais, 2002).

Dans le même esprit, d'autres études sont venues affirmer la primauté de l'expression locale et démontrer que le phénomène de la standardisation n'est pas aussi développé que certains veulent le faire croire: Britt 1974, Unwinn 1974, Ricks, Arpan et Fu 1974, Boddewyn. Soehl et Picard 1986, Mueller 1987, Hite et Frazer 1988, Synodinos, Keown et Jacobs 1989. Pour eux. la terre est une même planète avec ses diversités.

Pour Bille (1999, p.14). "Le mode de consommation du produit est également déterminant: le fromage par exemple se consomme avant le dessert en France, après le dessert en Grande Bretagne et au petit déjeuner en Hollande. Tous les films de publicité qui pourraient le mettre en scène doivent en tenir compte. (...) Le prime time est la chose la plus diverse que l'on puisse trouver : 18 heures en Allemagne; 18 heures ou 21 heures en Grande Bretagne : $20 \mathrm{~h} 30$ en France, 22 heures en Espagne ».

Les Américains sont devenus les champions de la mondialisation. Ils ont réussi à imposer leur culture, l'American Way of Life, leur langue, leur mode de vie comme étant des valeurs d'être en soi. Par la globalisation des produits et services, on se rend compte qu'on présente aux consommateurs des produits et des services qu'ils n'ont jamais demandés. La citrouille et l'Halloween sont apparus à la toute fin des années quatre-vingt-dix en France, après le Big Mac et le Coca Cola. Les Français avaient-ils vraiment besoin de cela pour améliorer leur qualité de vie? Martelart (1989), dans son Internationale publicitaire a bien expliqué le phénomène.

Pour contrer les accusations d'exploiteurs de la main d'oeuvre dans les pays en voie de développement, les grandes entreprises ont décidé d'exiger des conditions de travail dites équitables. Mais celles-ci sont en fait la transposition des normes occidentales du travail. Il importe davantage de satisfaire les consommateurs qui veulent se donner bonne conscience en achetant des objets produits selon leurs propres normes, que de se fondre dans les habitudes de la culture locale. On pratique sur le marché du travail, les mêmes travers que l'on a fait pour la religion.

Mais, lorsque les consommateurs occidentaux n'exercent aucune pression sur certains comportements de ces multinationales, celles-ci manifestent un mépris profond pour les populations exploitées. Les discours entourant les OGM témoignent de cette attitude (Fréchette, 1999). Pour les uns, c'est la solution au problème de la faim dans le monde. Pour les autres, c'est l'impérialisme des grandes sociétés qui tuent les pratiques agricoles ancestrales et le mode de vie social et culturel qui les entourent.

En Amérique et en Europe, la loi oblige les grands fabricants automobiles à doter leurs voitures de système antipollution. On vend ces mêmes voitures en Amérique latine, par exemple, sans ces systèmes parce qu'ils n'y sont pas contraints par la loi.

\section{Le monde de la culture}

23 Face à l'invasion de l'industrie culturelle américaine, les pays occidentaux ont adopté diverses mesures pour contrer les effets pervers d'une culture à sens unique. D'une part, nous assistons à travers le monde à une propension à l'uniformité. Alors qu'on prétend que le consommateur a le libre choix de ses goûts, les produits culturels auxquels il a accès lui sont imposés. Le cinéma américain, par son système de promotion et de distribution empêche les cinémas nationaux de plusieurs pays de s'épanouir. Pour les 
Américains, chacun peut mettre sur le marché ses idées et ses productions et si elles ne plaisent pas. il ne faut pas en vouloir aux Américains.

L'ouverture sur le monde est devenue une réalité de l'heure. Outre le fait qu'Internet accentue ces phénomènes, la radio avait déjà devancé ce problème et démontré la difficile limite de l'intégrité du territoire national. La bataille pour le contrôle des ondes dans les différents pays fut vite contournée par les émissions sur onde courte des grandes puissances. Et les règlements sur le contenu national à la radio, dans des pays comme le Canada et la France, protégeaient autant une industrie qu'une culture nationales. Il n'est venu à l'esprit d'aucun pays de protéger sa culture en limitant la diffusion d'œuvres classiques étrangères sur ses ondes. Au contraire, cette « intrusion » a toujours constitué une valeur ajoutée à la culture locale.

Par ailleurs, si la télé-série Dallas a été présentée dans de très nombreux pays du monde, elle n'a pas détrôné dans les cotes d'écoute les télé-séries locales. Le succès des téléromans québécois comme les telenovelas latino-américains en sont d'intéressants témoignages. La contradiction a été souvent soulevée : la globalisation est un concept qui véhicule son contraire, la segmentation (Robertson, 1992). Dans les pays occidentaux, les téléspectateurs ont accès, par le câble, à des dizaines de canaux de télévision; les satellites en proposent des centaines; la location de films vidéo, la possibilité d'enregistrement d'émissions, l'attrait d'Internet, les préoccupations et les goûts différents de chacun d'eux rendent caduque toute volonté de se fermer à la culture internationale.

Ce qui est en cause, ce n'est pas tant le nombre que l'omniprésence des grandes multinationales à tous les niveaux de la culture. En Amérique latine comme au Québec, que les trames des télé-séries soient nationales n'éliminent pas le fait que la télévision, dans sa conception, soit américaine et que les « soaps » soient de culture américaine.

Les réseaux d'informations continues sont orchestrées à partir des grandes capitales internationales et proposent un flux d'informations nord-sud. Mais cette invasion est aussi pondérée par les pratiques d'écoute locale de la télévision. Une enquête menée par l'Institut international des communications (IIC) de Londres et reprise par l'hebdomadaire britannique The Economist laisse entendre que loin d'être à l'heure américaine, les journaux télévisés dans le monde s'intéressent essentiellement... à leur propre pays, voire à leur propre patelin.

28 "L'IIC a fait une étude systématique des télé-journaux diffusés le soir du 19 novembre 1991 par 87 chaînes de télévision dans 55 pays. Les grandes nouvelles internationales du jour: l'intensification des combats en Yougoslavie, la libération de Terry Waite, le massacre au Timor oriental par les forces d'occupation indonésiennes et la «renomination » (bien éphémère) d'Edouard Chevardnadze au poste de ministre soviétique des Affaires étrangères.

29 « Hors d'Europe ce jour-là, l'affaire yougoslave n'a trouvé aucun écho. En dehors de l'Asie, pas un mot sur la tragédie de Timor... Mais la vraie vedette des bulletins, ce sont les reportages nationaux: le prix des œufs à Bangkok, les embouteillages à Rio, la famille royale en Grande Bretagne, etc.

30 "Selon l'IIC, le pourcentage de temps d'antenne accordé, durant les nouvelles télévisées, aux informations provenant de la partie du monde où se trouve la station émettrice, vont de 56 \% (en Océanie) à 92 pour cent (En Amérique latine) » (Brousseau, 1992). 


\section{L'exemple canadien}

31 Existe-t-il une culture canadienne? La question mérite d'être posée tellement est complexe la réalité canadienne. On y parle des peuples fondateurs, des sociétés distinctes, du multiculturalisme... (Dagenais, 1992). Lorsque l'on veut parler de culture canadienne, on fait face à un problème insoluble. Si le tango est argentin, la samba brésilienne, le flamenco espagnol, le Canada n'a pas de signature qui traduise son identité. En fait, le Canada constitue une énigme, car il n'y a pas une culture canadienne, il y a des cultures canadiennes. Or le drame ou le miracle canadien tout à la fois, c'est que ces différentes cultures ne se rencontrent jamais, mais se juxtaposent, s'opposent et se complètent. On loue la diversité canadienne comme un exemple positif de rencontres interculturelles, mais dans les faits, c'est l'hégémonie anglo-saxonne qui s'impose.

Le fait français : au moment de la conquête du Canada par les Anglais, en 1759, il n'y avait pratiquement pas d'anglophones au Canada. C'était un pays qui appartenait à la France, peuplé de Français et qui fut cédé à l'Angleterre dans la bataille qui opposait les deux pays pour l'hégémonie du monde. Le fait français va déranger les Anglais. Depuis près de 250 ans, les Canadiens anglais ont tout fait pour étouffer cette réalité. D'abord, ils ont essayé d'éliminer les Acadiens en les déportant tout simplement en Louisiane. Ils ont créé des institutions politiques où les francophones étaient minoritaires. Lord Durham écrivait en 1838 que le seul moyen de rendre les Québécois francophones heureux étaient de les assimiler à la majorité anglophone. Cette stratégie n'eut pas plus de succès que les autres. Puis on a trouvé l'artifice de la confédération. On a divisé le pays en dix : neuf provinces anglophones, une province francophone. Et on a laissé joué la loi de la démocratie : que la majorité l'emporte. Ne pouvant faire entendre leurs voix dans un tel contexte, les francophones du Québec se sont repliés sur leur gouvernement.

Mais loin d'affaiblir sa culture, cette situation a placé les francophones en état de continuelle autodéfense pour exister, pour s'affirmer, pour prospérer. Le Québec a su développer une culture propre. Il possède sa langue, sa religion, son droit civil, ses habitudes culinaires, son architecture propre, ses artistes sur la scène internationale, et ses chevaliers d'entreprises qui se sont implantés un peu partout à travers le monde. Mais la culture québécoise n'est pas la culture canadienne. Et la majorité des Anglophones du pays sont bien ennuyés de considérer que la culture canadienne est constituée en partie d'une culture et d'une langue qui leur sont étrangères.

34 Le fait anglais : 50 ans après la conquête du Canada, les Anglais sont désormais majoritaires au pays (Lachapelle. et Henripin, 1980, p. 11). Le pays en oublie ses racines françaises. Il se développe en anglais et il n'y a pas de place pour les francophones qui n'habitent pas la province de Québec. Leurs droits seront partout bafoués. La majorité de langue anglaise exige que la minorité vaincue s'adapte et elle affirme de façon nette son caractère et sa culture d'origine saxonne.

Mais toujours se dresse un mur infranchissable entre ses deux communautés de base : les francophones et les anglophones. L'écrivain canadien Hugh McLennan a utilisé l'expression suivante pour décrire le Canada: les deux solitudes. Le Canada se présente comme le lieu de développement de deux cultures distinctes et très fortes qui se sont développées parallèlement et, on peut même dire, en opposition. Les deux solitudes sont devenues les deux ennemis. Et ce faisant, ils ont créé une dynamique propre au pays. Si le 
Canada n'avait qu'à naviguer entre ces deux pôles, ce serait déjà un moindre mal. Mais voilà qu'apparaitt une troisième solitude, pour ne pas dire une troisième contrainte.

Le fait autochtone: occupées à gérer la difficile relation entre francophones et anglophones, les autorités avaient oublié qu'il existait toujours au Canada les descendants des premières nations autochtones qui revendiquaient aussi une place au soleil, après avoir été massacrés et dissipés par les blancs. Au fil des ans, le Canada les avait campés dans des réserves spécifiques un peu partout à travers le pays. On leur avait donné des droits et des territoires qu'on leur avait enlevé au fur et à mesure que changeaient les besoins de la nation canadienne.

Et puis on a voulu les aider, et avec grande générosité on a décidé de les éduquer. Ainsi, pendant des années, on a arraché à leur famille, les enfants autochtones pour leur apprendre la vie des villes dans lesquelles ils ont eu de la difficulté à s'adapter. Et en même temps, ils ont perdu leur savoir faire traditionnel de chasseurs et de pêcheurs. Mais ils se sont instruits et ils ont appris qu'on leur avait joué plusieurs vilains tours dans l'histoire. Et ils se sont mis à lire les traités, à les interpréter et à se rendre compte qu'on avait été somme toute peu généreux à leur endroit. On leur avait cédé toutes les terres incultes, inutiles, déboisées, arides et sans lendemain. Et surtout sans aucune voie de communication avec la civilisation. Mais voilà qu'on a voulu construire des barrages hydroélectriques dans les régions qui leur appartenaient. Les autochtones ont alors revendiqué des compensations pour l'utilisation de leurs terres, compensations qu'ils obtinrent. Et devant ces succès, ils se sont mis à exiger qu'on leur remette ce qu'on leur avait volé.

Voilà donc apparaître une troisième solitude qui réclame des droits, des pouvoirs, une autonomie et une reconnaissance et en même temps développe une culture forte et intéressante. Elle s'oppose aux deux autres solitudes qui tout en se sentant coupables des gestes du passé, sont davantage préoccupées à se définir une réalité qu'à partager avec les premières nations qui apparaissent comme des professionnels de la revendication. Mais cette solitude possède aussi une culture particulière, un art diversifié, l'Art Inuit chez les Esquimaux, l'art des Indiens de l'ouest et celui des Indiens des plaines. Ils ont apporté au pays la connaissance de la vie en hiver, des plantes médicinales. Ils ont aidé à construire les gratte-ciel puisqu'on a su exploiter leur absence de vertige.

Nous voilà donc dans un pays avec trois solitudes et chacune d'elles va donc apporter une contribution sérieuse à la culture canadienne. Et en même créer une dynamique d'affrontements. Le portrait serait relativement simple si on s'en tenait à ces trois solitudes bien campées. Mais voilà qu'il y en a une quatrième.

Le multiculturalisme: si le Canada est né du fait français, a été dominé par le fait anglais et doit reconnaitre l'existence du fait autochtone, aujourd'hui ce sont les immigrants de toutes les parties du inonde qui imposent leur présence. Actuellement, la proportion de la population se réclamant d'une origine ethnique autre que française et britannique approche les $40 \%$ de la population. Ils sont plus nombreux comme entité que les francophones du pays, que les anglophones et que les autochtones, pris séparément.

41 Ces immigrants ne partagent pas le poids du passé et ont décidé de venir vivre en Amérique où l'on retrouve un niveau de vie extraordinaire, la chance pour tous de réussir et la civilisation américaine. Ces nouveaux venus doivent s'intégrer dans un pays qui n'a jamais réussi à créer sa propre personnalité. Alors ils ont décidé de conserver la leur. Pour les autorités canadiennes, il était somme toute plus facile de considérer que le 
Canada était le mélange d'un nombre considérable de cultures plutôt que de reconnaître la réalité de ses deux peuples fondateurs.

Le Canada a décidé de préserver le multiculturalisme et a encouragé l'expression particulière des ethnies. Depuis 1971, le multiculturalisme devient une politique officielle du gouvernement qui prévoit même des programmes et des fonds pour les groupes ethniques. Mais en même temps que ces groupes viennent enrichir la culture canadienne, ils constituent une quatrième solitude qui essaie de faire sa place au milieu des trois autres.

Loin de creuser un «melting pot » comme aux Etats-Unis, le Canada va donc se diversifier de plus en plus. Et la culture canadienne devient le produit de ce mixage. Plus les solitudes se développent, plus la culture se complexifle. Mais alors qu'on croyait avoir terminé avec ces quatre solitudes, le véritable drame éclate pour la culture canadienne. La présence et la toute puissance des Américains détruit toute possibilité de créer une culture autonome.

Le fait américain : au début du XXe siècle, les Américains comptent 50 millions d'habitants ; le Canada 5 millions. En 1925, les États-Unis possèdent plus de 600 stations de radios; le Canada à peine 50. Ce rapport de force crée une situation extrêmement difficile pour la réalité canadienne. Les créateurs canadiens ne peuvent s'exprimer facilement parce que tout est fait aux États-Unis. Le Canada ne peut rivaliser avec ce voisin qui envahit tout le champ culturel de façon irrémédiable. Pour les Américains il n'existe qu'un seul continent. On produit les mêmes publicités; on reproduit le même mode de vie. On utilise les mêmes voitures et les mêmes produits. Et on se divertit et forge son imaginaire collectif à partir des mêmes films.

Les Américains ne comprennent pas ce qu'est la culture canadienne. Et les Canadiens consomment les produits culturels américains comme si c'était des produits locaux tellement la culture des deux peuples se ressemblent. L'utilisation d'une langue commune, la pratique d'habitudes communes, rapprochent les deux communautés nordaméricaines. Encore une fois, c'est sous le coup d'une attaque de l'extérieur que le Canada va réagir. Devant cet envahissement américain, devant la difficulté pour ses créateurs de s'exprimer, le Canada va donc revendiquer le droit à sa culture nationale.

Les Américains ne veulent rien entendre. Pour eux la culture est universelle, c'est-à-dire américaine. Alors le Canada insiste. Et encore aujourd'hui la question n'est pas réglée. Un des problèmes de l'accord du libre échange entre le Canada et les États-Unis, en 1992, fut de protéger les industries culturelles canadiennes. Pour les Américains, il s'agit là d'un commerce qui ne doit pas être réglementé. Pour les Canadiens, il s'agit là d'un volet essentiel à sa culture. L'ambassadeur américain au Canada, Thomas Niles, disait alors : "Nous croyons que ces questions devraient être abordées selon un strict point de vue commercial et que les gouvernements ne devraient pas se mêler de cela (Simpson, 1985).

47 Le représentant américain dans l'accord du libre échange canado-américain, Clayton Yeutter, a eu cette parole bien significative : « Je suis prêt à mettre la culture américaine sur la table des négociations et accepter de subir tous les inconvénients que cela pourrait lui occasionner par la culture canadienne. J'espère que le Canada est prêt de son côté à prendre le même risque » (Lewington, 1987).

Le fait québécois: au Québec, les cinq millions de francophones sont entourés, pour ne pas dire asphyxiés culturellement, par plus de 300 millions d'anglophones. Le marché nord- 
américain constitue un seul et même tout. De ce fait, on ignore la diversité culturelle des francophones d'Amérique. La culture francophone doit donc apprendre à s'affirmer dans cette cacophonie. En fait, la diversité culturelle au Canada n'existe qu'autant qu'elle protège les acquis des anglophones (Dagenais, 1989). A vouloir tout niveler, le concept de mondialisation retrouve au Canada ses effets pervers et contre productif., car ce qui fonctionne, c'est la différence.

\section{Un discours d'imposture ou d'ouverture}

Le discours sur les bienfaits et les méfaits de la communication transnationale est donc un discours teinté a priori des intérêts et de l'idéologie de celui qui le pratique ou qui en discute. La querelle sur les effets positifs et négatifs de cette mondialisation en témoigne (Featherstone et al, 1995). Pour les tenants de la libre circulation des biens et services, apporter aux peuples de la terre les produits de la civilisation avancée, c'est leur faire partager les progrès de l'humanité. Pour ceux qui subissent cet apport « humanitaire », il s'agit d'une invasion. Par ailleurs, leurs discours de victimes cachent parfois des intérêts économiques, parfois des intérêts politiques et parfois alimentent aussi des préoccupations sincères sur l'intégrité de la culture. Mais trop souvent, même si le discours des organisations insiste pour démontrer qu'elles s'ouvrent sur la culture des autres, dans les faits, elles la méprisent et l'exemple du Canada en témoigne.

Featherstone (1995) et tant d'autres chercheurs ont démontré qu'il ne fallait pas perdre de vue que la mondialisation était accompagnée, par un effet de retour de balancier, du développement des identités locales. Les discours sur la mondialisation sont trop souvent des discours de rétrécissement. Or la mondialisation apporte aussi des éléments d'ouverture et de changements positifs.

Giono est devenu un écrivain international en décrivant son petit coin de pays. Marquez a remporté le prix Nobel de la littérature en traduisant de façon merveilleuse l'âme latinoaméricaine. Dans leurs cas, ce qui fait l'internationalisme, c'est justement l'affirmation de la différence et non la recherche de l'homogénéisation. Le drame de la globalisation, c'est toutefois que les grandes entreprises et les grandes puissances cherchent à niveler la différence, croyant y trouver une solution à leur développement économique alors qu'ils sont probablement en train de le bloquer.

La mondialisation peut emprunter plusieurs avenues: donner à chacun ce qu'il recherche, imposer à tout le monde ce qui ne lui convient pas ou un mélange de tout cela. Lorsque McLuhan (1951) a traité du village global pour la première fois dans son texte intitulé Front Page, il voulait signifier que sur la première page d'un journal, le monde entier se déroulait sous les yeux du lecteur. On a toutefois vite fait de renverser cette approche pour lui faire dire l'inverse. Le village global ne signifiait pas pour lui la création de l'homme unidimensionnel, mais plutôt l'ouverture de chacun à l'autre, sans perdre son identité. 


\section{BIBLIOGRAPHIE}

AFP, La pub : chacun son style. Montréal, La Presse, 1989, 9 août, p 4. BELK, R. W. et Richard W. Pollay, Materialism and Status Appeals in Japanese and U.S. Print Advertising, International Marketing Review 2, 4, 1985, pp. 38-47.

BENEDICT, R., Patterns of Culture. New York: Mentor Book, 1959, 255 p. BILLE, J., À la recherche du consommateur européen. Revue Market Management. Paris: Ed. Eska, no 3, 1999, pp. 10-15

BODDEWYN. J.. R. S. et J. PICARD, Standardization in International Marketing: Is Ted Levitt in Fact Right? Business Horizons 29, 6, 1986, pp. 69-75.

BRITT, S.H., Standardizing Marketing for the International Market, Columbia Journal of World Business, 1974, 9,4, pp. 39-45

BROUSSEAU. F., Le succès des idées fausses, Le magazine 30, mai 1992, p. 23.

DAGENAIS, B., La publicité au Québec ou comment échapper à 250 millions d'anglophones, in HOTIER H. (Éd.), Cultures d'entreprise et communication internationale. Bordeaux : GREC/O. 1989.

DAGENAIS. B., La culture canadienne ou la rencontre des solitudes.

Communication présentée aux journées culturelles, Encuentro de dos

continentes, Tucuman, Argentine, le 5 novembre 1992, 9 p.

DAGENAIS, B.. La publicité et les relations publiques, partenaires essentiels de la mondialisation, in La communication internationale : mondialisation, acteurs et territoires socioculturels, sous la direction de Gilles Brunei et Claude-Yves Charron, Boucherville : Gaétan Morin, 2002, pp. 143-151.

D’IRIBARNE. P., La logique de l'honneur. Paris : Éditions du Seuil, 1989,

279 p., cité dans Hotier 2001.

DUNN, S. Watson, The Case Study Approach in Cross-Cultural Research. Journal of Marketing Research 3.1, 1966. pp. 26-31.

ELINDER. E., How International Can European Advertising Be? Journal of Marketing 29, 2, 1965 , pp. 7-11.

FATT. A., The Danger of Local International Advertising, Journal of Marketing 31,1, 1967 pp. 60-62.

FEATHERSTONE, M.. S. LASH ET R. ROBERTSON (Éd.), Global Modemilies. Londres: Sage Publications, 1995, $292 \mathrm{p}$.

FIELDS, G., Fulfilling an Eastern Fantasy: How Western Stars from Bronson to McEnroe Enchant Japanese Viewers and Move Products, Ach'ertising Age, 8 mai 1989.

FRECHETTE, K., Aliments transgéniques : jeux de pouvoir et contrôle de l'information. Essai de maîtrise en Relations internationales. Université Laval, 1999,51 p.

HITE, R. et C. FRAZER, International Advertising Strategies of Multinational Corporations, Journal of Advertising Research, 28,4, 1988, pp. 9-17. HOT1ER, H., Les cultures nationales face aux 
entreprises transnationales, in Lihua Zheng et Hua XUZE (éd). Entreprise et communication, Hong Kong : Éditions Quaille. 2001.

IZRAELEWICZ, E.. Ce n'est pas la culture américaine qui s'impose, mais une culture globale. Le Monde, 11 mars 1997, p. 17.

KANSO, A., International Advertising Strategies: Global Commitment to Local Vision, Journal of Advertising Research, 32. 1. 1992, pp. 10-14. LACHAPELLE, R. et HENRIPIN J.. La situation démolinguistique au Canada : évolution passée et prospective, Montréal : Institut de recherches politiques, 1980, $391 \mathrm{p}$.

LEVITT, T.. The Globalization of Markets, Harvard Business Review 61,3, 1983, pp. 92-102.

LEWINGTON, J., Fuzzy Words Blur Culture Debate. Toronto, Globe and Mail. Février 5, 1987, in SMITH A., Canadian Culture, the Canadian State, and the New Continentalism, Canadian-American Public Policy, nov. 3, oct 1990, 36 p. MARQUEZ, F.T., Cross Cultural Research: A Decision Factor in Standardized versus Non-Standardized Global Advertising, Gazette 25, 3, 1979, pp. 150-162. MATTELART, A., L'internationale publicitaire. Paris : Éditions de la découverte. 1989, 250 p.

MCLUHAN, M., The Mechanical Bride: The Folklore of Industrial Man, New York: Vanguard Press, 1951.

MUELLER. B.. Retlections of Culture: An Analysis of Japanese and American Advertising Appeals, Journal of Advertising Research, 27. 1987, pp. 51 -59. MUELLER. B.. Standardization vs Specialisation: An examination of Westernization in Japanese Advertising, Journal of Advertising Research, 32, 1, 1992, pp. $15-25$

RICKS. D., J. ARPAN et M. FU. Pitfalls in Advertising Overseas, Journal of Advertising Research 14, 6, 1974, pp. 47-51.

ROBERTSON, R.. Globalization: Social Theory and Global Culture. Londres: Sage publications, 1992, $212 \mathrm{p}$.

ROSENBERG. L., Deciphering the Japanese Cultural Code. International Marketing Review, 3, 3, 1986, pp. 47-57.

RUTIGLIANO, A., The Debate Goes On: Global vs. Local Advertising. Management Review, 75, 6, 1986, pp. 27-31.

SEKIGAHAMA, R., Madison Avenue Goes Shinjuku, AsiAm, janvier 1988. SIMPSON, J., Sovereign Principles, Toronto, Globe and Mail, novembre 2014, 1985, in SMITH A., Canadian Culture, the Canadian State, and the New Continentalism. Canadian-American Public Policy, nov. 3. oct 1990. 36 p. SORENSON, R. et U. WIECHMANN, How Multinational View Marketing Standardization, Harvard Business Review 53, 3, 1975, pp. 38-44 et 48-50. SYNODINOS, N.. C. KEOWN et L. JACOBS. Transitional Advertising Practices: A Survey of Leading Brand Advertisers. Journal of Advertising Research 29. 2, 1989, pp. 43-50.

UNWINN, S.. How Culture affects Advertising Expression and Communication Style, Journal of Advertising, 3, 2, 1974, pp. 24-27.

YOSHINO, M.Y., Marketing in Japan: A Management Guide, New York: Praeger, 1975.

\section{RÉSUMÉS}

L'histoire des grandes religions et des grandes civilisations démontre que la mondialisation et ses effets pervers a toujours existé. Aujourd'hui, celle-ci s'accompagne d'un triple discours. Celui des Américains, qui tentent de faire fi de la diversité culturelle pour mieux imposer leurs produits et 
leur culture. Celui des États qui veulent protéger leur identité et leur industrie culturelles. Et celui de ces mêmes États qui refusent de reconnaître sur leur propre territoire toute diversité qui viendrait déranger la majorité. Mais ces discours cachent aussi des guerres d'intérêts.

The history of the main religions and of the great civilizations of the world has taught us that the globalization and its side effects have existed since long ago. Today, though, the discourses on the phenomena have changed. On the one hand, the Americains try to ignore any cultural diversity so their products and culture can get anywhere. On the other hand, any state try to protect his own cultural industry and identity. And at last, these same states will, inside their borders be very slow to accept the diversity of culture. But all those different discourses hide one main object: the prosperity of the economy of any state.

\section{INDEX}

Mots-clés : diversité culturelle, globalisation, guerre d'intérêts, religion

\section{AUTEUR \\ BERNARD DAGENAIS}

Bernard Dagenais est professeur titulaire au Département d'information et de communication de l'Université Laval, à Québec. Il est également président du Conseil d'administration de l'Agence Préambule Communication. 